\begin{abstract}
Iranica
Abstracta Iranica Revue bibliographique pour le domaine irano-aryen

Volume 34-35-36 | 2017

Comptes rendus des publications de 2011-2013
\end{abstract}

\title{
Hamid Naficy. A Social History of Iranian Cinema
}

\section{Agnès Devictor}

\section{OpenEdition}

Journals

Édition électronique

URL : http://journals.openedition.org/abstractairanica/42407

DOI : 10.4000/abstractairanica.42407

ISSN : 1961-960X

Éditeur :

CNRS (UMR 7528 Mondes iraniens et indiens), Éditions de l'IFRI

\section{Référence électronique}

Agnès Devictor, "Hamid Naficy. A Social History of Iranian Cinema », Abstracta Iranica [En ligne], Volume 34-35-36 | 2017, document 2, mis en ligne le 30 juillet 2017, consulté le 28 septembre 2020. URL :

http://journals.openedition.org/abstractairanica/42407; DOI : https://doi.org/10.4000/

abstractairanica.42407

Ce document a été généré automatiquement le 28 septembre 2020.

Tous droits réservés 


\title{
Hamid Naficy. A Social History of Iranian Cinema
}

\author{
Agnès Devictor
}

\section{RÉFÉRENCE}

Hamid Naficy. A Social History of Iranian Cinema. Durham and London, Duke University Press, 2011-2012, 4 volumes (Volume 1: The Artisanal Era, 1897-1941, 389 p.; Volume 2: The Industrializing Years, 1941-1978, 526 p.; Volume 3: The Islamicate Period, 1978-1984, 256 p.; Volume 4: The Globalizing Era, 1984-2010, 632 p.)

1 Hamid Naficy, l'un des meilleurs connaisseurs du cinéma iranien et surtout très fin analyste de ses enjeux historiques, politiques, sociaux, et industriels signe une somme inégalée, fruit de trente années de recherches et qui n'a aucun équivalent dans les études publiées en Occident sur le cinéma iranien. Ce travail d'érudition déborde amplement l'étude en histoire sociale du cinéma pour analyser aussi les mutations de l'Iran à l'ère de la modernisation industrielle et le rapport que l'Etat iranien autoritaire (quels que soient les époques et les dirigeants) a entretenu avec le cinéma depuis son apparition jusqu'à aujourd'hui. Hamid Naficy porte une attention toute particulière à la question des luttes d'influence diplomatiques qui ont pesé sur le cinéma iranien depuis l'élaboration d'une industrie nationale, mais aussi à l'enjeu politique qu'est devenu le cinéma dans la politique extérieure de l'Iran. Il prolonge cette analyse en montrant combien, à partir de 1979, la création cinématographique hors d'Iran, du fait d'une diaspora dense et dynamique, est devenue aussi une prolongation de ce cinéma iranien dans un contexte de mondialisation.

2 Les quatre volumes qui composent cet ouvrage et qui traitent chacun d'une période spécifique peuvent être lus séparément tant ils possèdent une cohérence interne.

3 Le volume 1 s'attache à l'importation de la technique cinématographique et à son adoption comme pratique sociale et comme nouveau champ de création à la fin de l'ère Qadjar et à l'époque de la modernisation autoritaire de Reḍā Šāh. Naficy rappelle que le 
cinéma se répand à l'heure de l'industrialisation, de l'accélération du temps et de la réduction des distances et que le cinéma participe indéniablement, en Iran comme ailleurs, d'un processus d'individualisation. Dans la société iranienne traditionnelle, le film est une adresse à soi, personnelle, qui joue sur l'intime, définissant aussi de nouveaux comportements dans l'espace de la salle de cinéma. L'A., qui puise notamment dans les récits de voyage et dans l'iconographie de l'époque une masse d'informations colorées, parvient à retracer l'ambiance des séances de cinéma avec leur public masculin et indiscipliné. L'homme qui y commentait pour l'assistance les films en provenance de l'étranger finissait d'ailleurs par se servir de sa canne pour taper sur la tête des spectateurs les plus dissipés et tenter ainsi de les faire taire! Naficy analyse comment, à l'époque de Reḍa Šāh, le cinéma a été l'une des pièces du programme de modernisation et de sécularisation de l'Iran multiethnique pour aboutir à un Etat Nation homogène et centralisé. Un processus de contrôle a ainsi encadré de façon minutieuse le cinéma, qui a dû s'employer à montrer des représentations de cette modernisation, pour la rendre désirable et ainsi l'accélérer. Sur les écrans, les femmes dévoilées et les personnages de dandy ont contribué à faire des films un agent de ce profond changement. Mais durant toute cette période, où les influences étrangères (et notamment celles de l'Inde) restaient puissantes, le cinéma iranien n'a pas dépassé le stade de l'artisanat.

Le volume 2 est consacré à la période du règne de Moḥammad Reḍā Šāh Pahlavī au cours de laquelle le cinéma est entré dans un processus industriel, produisant près de 90 films par an, toujours très encadrés par une censure efficace. Par ailleurs, dès les lendemains de la Seconde Guerre mondiale, Naficy pointe que les écrans de cinéma étaient devenus un enjeu d'influence pour les trois grandes puissances (la GrandeBretagne, les Etats-Unis et l'Union Soviétique) qui se disputaient l'Iran. Il s'agissait par exemple pour les Etats-Unis non seulement de distribuer les films de Hollywood, notamment au moyen d'unités mobiles, mais de savoir aussi précisément ce que les Iraniens voyaient sur les écrans (avec l'aide d'espions), pour trouver ainsi les moyens d'encadrer l'imaginaire collectif. Ces années furent celles de l'émergence du filmfärsī, genre national très populaire qui domina les écrans et dont Naficy analyse les codes, les stars et les effets sur la société. C'est aussi durant les années 60 et 70 qu'un cinéma moderne, une Nouvelle Vague à l'iranienne, a posé un regard aiguisé sur l'évolution politique et sociale de l'Iran avec des films d'une grande rigueur esthétique. Paradoxalement, ces films, très critiques envers le pouvoir, étaient financés et aussi censurés par l'Etat. En relation avec l'étranger, ils ont activement participé à ce que Naficy étudie avec attention : un cinéma iranien qui déjà dépassait ses frontières. C'est aussi à cette époque et au sein de ces mouvements esthétiques novateurs qu'une culture underground s'est développée, terreau fertile des racines de la future révolution.

Le volume 3 concerne l'année de la révolution (particulièrement destructrice pour les salles de cinéma), l'islamisation du régime, et la politique volontariste de reconversion de l'industrie cinématographique pour la décharger de tout « ferment de corruption morale ». Institutionnalisation, financement, mais aussi contrôle du cinéma s'articulent à partir de 1982 pour mettre en place un cinéma en conformité avec le nouveau régime. Durant cette période post-révolutionnaire, c'est à dire aussi durant les huit années de guerre, le cinéma qui a subi un contrôle des mœurs inégalé dans l'histoire a aussi été un puissant levier dans la construction d'une représentation nationale. La culture née de 
ce mouvement d'islamisation n'a cependant jamais été homogène ni statique comme le montre avec force détails Naficy.

Dans le volume 4, l'A. présente l'effort de guerre pris en charge par le cinéma, aussi bien documentaire que fiction. Hamid Naficy souligne combien, à partir de ces années, la forme documentaire ne s'est plus limitée à l'enregistrement d'événements pour prendre toute son autonomie formelle et sa puissance critique. Des réalisateurs ont ainsi continué d'explorer ce genre en empruntant des voies variées, du côté du cinéma ethnographique, des cultures émergeantes et des phénomènes de société (avec par exemple des films sur les nez refaits ou sur la passion du football), tout en poursuivant la longue tradition des documentaires sur les pratiques artistiques en Iran. Mais surtout, le cinéma documentaire s'est frotté avec ténacité à des sujets brûlants, non pas implicitement comme l'a fait le cinéma de fiction qui use de stratégies d'évitement, de sous-entendus et de métaphores pour aborder des questions politiques ou sociales bannies des écrans, mais en les traitant très explicitement. Nombre de ces films ont été frappés par la censure, mais ce regard critique sur la société ne s'est pas détourné pour autant. Dans un autre chapitre, Naficy met en lumière l'affirmation d'une présence féminine devant et derrière la caméra à partir du milieu des années 1980 et montre comment le foulard, instrument d'oppression, a été détourné par les cinéastes dans des formes esthétiques très innovantes pour mettre en crise cette pratique sociale et politique. L'A. achève ce volume particulièrement dense par deux chapitres qui constituent sans doute l'apport le plus singulier à cette longue histoire du cinéma iranien. Le chapitre consacré à une nouvelle diplomatie d'influence (new public diplomacy) explore les territoires sur lesquels se déroulent toujours des luttes sans mercis entre l'Iran et les Etats-Unis. Il souligne que sous les présidences Huātamī et Aḥmadīnežād, les questions liées au cinéma et aux médias ont rejoint ces formes de diplomatie, truffées de paradoxes et où les différents gouvernements iraniens, tout en étant en lutte contre les influences étrangères notamment venues de la diaspora, ont déployé aussi une politique très offensive destinée autant à leur propre territoire qu'à une influence très large hors de ses frontières. Enfin, suite à la répression qui a suivi les élections de 2009, un cinéma underground utilisant Internet pour sa diffusion s'est développé en réponse au musellement imposé à toute forme d'expression en Iran. Des formes cinématographiques ont alors tenté de rendre compte d'un exil intérieur vécu par bien des cinéastes, dont Ja'far Panāhī est devenu le symbole, exil interne qui a pu " être étouffant ou régénérant ". Dans ce contexte, le désir de quitter l'Iran n'a fait qu'augmenter, et le territoire iranien a ainsi continué à se vider d'une partie de ses forces vives cinématographiques qui ont gagné d'autres territoires de création, alimentant cet accented cinema tel que Naficy l'a défini dans un précédent ouvrage portant ce titre, et auquel est consacré le dernier chapitre.

7 Particulièrement bien référencé, comportant une masse inédite d'informations, loin de la littérature partisane ou de la vulgate sur le cinéma iranien, ce livre en quatre volumes est un outil indispensable pour qui s'intéresse au cinéma mais plus généralement à cette région du monde ainsi qu'aux logiques d'influence qui marquent l'ouverture de ce nouveau siècle. 


\section{AUTEURS}

AGNĖS DEVICTOR

Université Panthéon Sorbonne 